\title{
SOBOLEV ORTHOGONAL POLYNOMIALS AND SPECTRAL DIFFERENTIAL EQUATIONS
}

\author{
I. H. JUNG, K. H. KWON, D. W. LEE, AND L. L. LITTLEJOHN
}

Dedicated to the memory of Professor H. L. Krall

Abstract. We find necessary and sufficient conditions for a spectral differential equation

$$
L_{N}[y](x)=\sum_{i=1}^{N} \ell_{i}(x) y^{(i)}(x)=\lambda_{n} y(x)
$$

to have Sobolev orthogonal polynomials of solutions, which are orthogonal relative to the Sobolev (pseudo-) inner product

$$
\phi(p, q)=\int_{\mathbf{R}} p q d \mu+\int_{\mathbf{R}} p^{\prime} q^{\prime} d \nu,
$$

where $d \mu$ and $d \nu$ are signed Borel measures having finite moments. This result generalizes a result by $\mathrm{H}$. L. Krall, which handles the case when $d \nu=0$.

\section{INTRODUCTION}

We consider a linear differential equation of order $N \geq 1$ of the spectral type

$$
L_{N}[y](x)=\sum_{i=1}^{N} \ell_{i}(x) y^{(i)}(x)=\lambda_{n} y(x),
$$

where each $\ell_{i}(x)=\sum_{j=0}^{i} \ell_{i j} x^{j}$ is a polynomial of degree $\leq i$, independent of $n=0,1, \cdots, \ell_{N}(x) \not \equiv 0$, and $\lambda_{n}$ are eigenvalue parameters given by

$$
\lambda_{n}=\ell_{11} n+\ell_{22} n(n-1)+\cdots+\ell_{N N} n(n-1) \cdots(n-N+1) .
$$

Our purpose is to find conditions for the differential equation (1.1) to have Sobolev orthogonal polynomials of solutions, which are orthogonal relative to some Sobolev (pseudo-) inner product of the form

$$
\phi(p(x), q(x))=\int_{\mathbb{R}} p(x) q(x) d \mu(x)+\int_{\mathbb{R}} p^{\prime}(x) q^{\prime}(x) d \nu(x),
$$

where $d \mu(x)$ and $d \nu(x)$ are signed Borel measures on the real line $\mathbb{R}$ with finite moments.

Received by the editors August 12, 1994 and, in revised form, December 7, 1994.

1980 Mathematics Subject Classification (1985 Revision). Primary 33C45.

Key words and phrases. Sobolev orthogonal polynomials, Sobolev bilinear forms, spectral differential equations. 
When $d \nu(x)=0$, such conditions were already found by H. L. Krall [6] (see also [10] for another proof of Krall's theorem).

Recently, R. Koekoek and H. G. Meijer [5] introduced a new class of Sobolev orthogonal polynomials, $\left\{L_{n}^{\alpha, M, N}(x)\right\}_{n=0}^{\infty}$, called the generalized Laguerre polynomials, which are orthogonal relative to the Sobolev inner product

$$
\frac{1}{\Gamma(\alpha+1)} \int_{0}^{\infty} p(x) q(x) x^{\alpha} e^{-x} d x+M p(0) q(0)+N p^{\prime}(0) q^{\prime}(0),
$$

where $\alpha>-1, M \geq 0$, and $N \geq 0$. On the other hand, R. Koekoek [3, 4] found an infinite-order differential equation with polynomial coefficients, which has $\left\{L_{n}^{\alpha, M . N}(x)\right\}_{n=0}^{\infty}$ as solutions. As a special case, he also found differential equations of the form (1.1) of order $\leq 4 \alpha+10$, which have $\left\{L_{n}^{\alpha, M, N}(x)\right\}_{n=0}^{\infty}$ as solutions for $\alpha=0,1,2$. Note that the inner product (1.4) is exactly of type (1.3).

These examples motivate the present work and the work by Kwon and Littlejohn [9], in which the special case with $N=2$ is handled.

Our main result (see Theorem 3.7 below) generalizes Krall's theorem (see Theorem 2 in [6] and Theorem 2.4 in [10]), which handles a special case with $d \nu(x)=0$ and a result in [9] (see Theorem 4.1 in [9]), which handles a special case with $N \leq 2$.

\section{Preliminaries}

All polynomials throughout this work are assumed to be real polynomials of a real variable $x$; the space of all such polynomials is denoted by $\mathscr{P}$. We shall denote the degree of a polynomial $\pi \in \mathscr{P}$ by $\operatorname{deg}(\pi)$, with the convention that $\operatorname{deg}(0)=-1$. By a polynomial system (PS), we mean a sequence of polynomials $\left\{\phi_{n}(x)\right\}_{n=0}^{\infty}$ with $\operatorname{deg}\left(\phi_{n}\right)=n(n \geq 0)$; in this case $\left\{\phi_{n}(x)\right\}_{n=0}^{\infty}$ is a basis for $\mathscr{P}$. We call any linear functional $\sigma: \mathscr{P} \rightarrow \mathbb{R}$ a moment functional and denote its action on a polynomial $\pi$ by $\langle\sigma, \pi\rangle$. With this action, any moment functional $\sigma$ defines a symmetric bilinear form on $\mathscr{P} \times \mathscr{P}$ by the formula $\langle\sigma, p q\rangle(p, q \in \mathscr{P})$.

We say that a moment functional $\sigma$ is quasi-definite (respectively, positivedefinite) if the moments

$$
\sigma_{n}:=\left\langle\sigma, x^{n}\right\rangle \quad(n \geq 0)
$$

of $\sigma$ satisfy the Hamburger condition

$$
\left.\Delta_{n}(\sigma):=\operatorname{det}\left[\sigma_{i+j}\right]_{i, j=0}^{n} \neq 0 \quad \text { (respectively, } \Delta_{n}(\sigma)>0\right)
$$

for each $n \geq 0$.

More generally for any symmetric bilinear form $\phi(\cdot, \cdot)$ on $\mathscr{P} \times \mathscr{P}$, we call the double sequence

$$
\phi_{m, n}:=\phi\left(x^{m}, x^{n}\right) \quad(m \text { and } n \geq 0)
$$

the moments of $\phi(\cdot, \cdot)$ and say that $\phi(\cdot, \cdot)$ is quasi-definite (respectively, positive-definite) if

$$
\Delta_{n}(\phi):=\operatorname{det}\left[\phi_{i, j}\right]_{i, j=0}^{n} \neq 0 \quad\left(\text { respectively, } \Delta_{n}(\phi)>0\right)
$$

for each $n \geq 0$. 
Lemma 2.1. A symmetric bilinear form $\phi(\cdot, \cdot)$ on $\mathscr{P} \times \mathscr{P}$ is quasi-definite (respectively, positive-definite) if and only if there are $P S\left\{P_{n}(x)\right\}_{n=0}^{\infty}$ and real constants $K_{n} \neq 0$ (respectively, $K_{n}>0$ ) for $n \geq 0$ such that

$$
\phi\left(P_{m}(x), P_{n}(x)\right)=K_{n} \delta_{m n} \quad(m \text { and } n \geq 0) .
$$

Moreover, in this case, the PS $\left\{P_{n}(x)\right\}_{n=0}^{\infty}$ satisfying (2.3) is uniquely determined up to non-zero constant multiples.

Proof. Assume that $\phi(\cdot, \cdot)$ is quasi-definite. Define a sequence of polynomials by

$$
P_{0}(x):=1
$$

and

$$
P_{n}(x):=\left[\Delta_{n-1}(\phi)\right]^{-1} \operatorname{det}\left[\begin{array}{cccc}
\phi_{0,0} & \phi_{0,1} & \ldots & \phi_{0, n} \\
\phi_{1,0} & \phi_{1,1} & \ldots & \phi_{1, n} \\
\vdots & \vdots & \ddots & \vdots \\
\phi_{n-1,0} & \phi_{n-1,1} & \ldots & \phi_{n-1, n} \\
1 & x & \ldots & x^{n}
\end{array}\right] \quad(n \geq 1)
$$

Then $\left\{P_{n}(x)\right\}_{n=0}^{\infty}$ is a monic PS and we have (2.3) with

$$
K_{n}=\Delta_{n}(\phi) / \Delta_{n-1}(\phi) \quad(n \geq 0),
$$

where $\Delta_{-1}(\phi)=1$. Now let $\left\{\widetilde{P}_{n}(x)\right\}_{n=0}^{\infty}$ be another PS satisfying

$$
\phi\left(\widetilde{P}_{m}, \widetilde{P}_{n}\right)=\widetilde{K}_{n} \delta_{m n} \quad(m \text { and } n \geq 0),
$$

where $\widetilde{K}_{n} \neq 0$. Since $\left\{P_{n}(x)\right\}_{n=0}^{\infty}$ is a PS, for each $n \geq 0$ we may write $\widetilde{P}_{n}(x)$ as

$$
\widetilde{P}_{n}(x)=\sum_{j=0}^{n} C_{n, j} P_{j}(x)
$$

for some real constants $C_{n, j}(j=0,1, \ldots, n)$ with $C_{n, n} \neq 0$. Then, for any $k=0,1, \ldots, n-1$, we have, by the orthogonality (2.3),

$$
0=\phi\left(\widetilde{P}_{n}, P_{k}\right)=\sum_{j=0}^{n} C_{n, j} \phi\left(P_{j}, P_{k}\right)=\sum_{j=0}^{N} C_{n, j} K_{j} \delta_{j k}=C_{n, k} K_{k}
$$

so that $C_{n, k}=0$. Hence we have $\widetilde{P}_{n}(x)=C_{n, n} P_{n}(x)$. Conversely, assume that there is a PS $\left\{P_{n}(x)\right\}_{n=0}^{\infty}$ satisfying (2.3). We may assume that each $P_{n}(x)$ is monic so that $\left\{P_{n}(x)\right\}_{n=0}^{\infty}$ is the unique monic PS satisfying (2.3). Writing $P_{n}(x)=\sum_{k=0}^{n} C_{k}^{n} x^{k}\left(C_{n}^{n}=1\right)$, we see that the condition (2.3) is equivalent to

$$
\sum_{k=0}^{n} \phi_{m, k} C_{k}^{n}=\phi\left(x^{m}, P_{n}(x)\right)=\phi\left(P_{m}(x), P_{n}(x)\right)=K_{n} \delta_{m n}
$$

for $n \geq 0$ and $m=0,1, \ldots, n$. Since for any fixed $n \geq 0$ the simultaneous equations (2.6) have a unique non-trivial solution $\left\{C_{k}^{n}\right\}_{k=0}^{n}$, we have $\Delta_{n}(\phi) \neq 0$ $(n \geq 0)$. Finally, we have that $K_{n}>0$ for $n \geq 0$ if and only if $\Delta_{n}(\phi)>0$ for $n \geq 0$. 
By various representation results like Boas' moment theorem [1] or Duran's generalization [2] of Boas' Theorem, any moment functional $\sigma$ will have an integral representation of the form

$$
\langle\sigma, \pi(x)\rangle=\int_{\mathbb{R}} \pi(x) d \mu(x) \quad(\pi(x) \in \mathscr{P})
$$

or

$$
\langle\sigma, \pi(x)\rangle=\int_{\mathbb{R}} \pi(x) \psi(x) d x \quad(\pi(x) \in \mathscr{P}),
$$

where $\mu(x)$ is a function of bounded variation on $\mathbb{R}$ (so that $d \mu$ is, in general, a signed Borel measure on $\mathbb{R}$ ) and where $\psi(x)$ is a $C^{\infty}$-function of the Schwartz class. With these representations, the Sobolev inner product given in (1.3) can be written as

$$
\phi(p(x), q(x))=\langle\sigma, p(x) q(x)\rangle+\left\langle\tau, p^{\prime}(x) q^{\prime}(x)\right\rangle,
$$

where $\sigma$ and $\tau$ are moment functionals. As we shall see, it is more advantageous for us to use this abstract notation involving moment functionals instead of using one of the above integral representations of moment functionals.

In case the symmetric bilinear form $\phi(\cdot, \cdot)$ in $(2.7)$ is quasi-definite, we call a corresponding PS $\left\{P_{n}(x)\right\}_{n=0}^{\infty}$ in Lemma 2.1 a Sobolev orthogonal polynomial system (SOPS) relative to $\phi(\cdot, \cdot)$. When $\tau=0$, we simply call $\left\{P_{n}(x)\right\}_{n=0}^{\infty}$ an orthogonal polynomial system (OPS) relative to $\sigma$.

Since a PS is a basis for $\mathscr{P}$, any PS $\left\{\phi_{n}(x)\right\}_{n=0}^{\infty}$ determines a moment functional $\sigma$ (uniquely up to a non-zero constant multiple), called a canonical moment functional for $\left\{\phi_{n}(x)\right\}_{n=0}^{\infty}$, by the conditions

$$
\left\langle\sigma, \phi_{0}(x)\right\rangle \neq 0 \text { and }\left\langle\sigma, \phi_{n}(x)\right\rangle=0 \quad(n \geq 1) .
$$

Remark 2.1. Suppose that the symmetric bilinear form $\phi(\cdot, \cdot)$ in $(2.7)$ is quasidefinite, and let $\left\{P_{n}(x)\right\}_{n=0}^{\infty}$ be an SOPS relative to $\phi(\cdot, \cdot)$. Then $\sigma$ must be a canonical moment functional for $\left\{P_{n}(x)\right\}_{n=0}^{\infty}$. In particular, $\langle\sigma, 1\rangle \neq 0$.

Now we introduce some formal calculus on moment functionals. For a moment functional $\sigma$ and a polynomial $\pi(x)$, we let $\sigma^{\prime}$, the derivative of $\sigma$, and $\pi(x) \sigma$, the multiplication of $\sigma$ by a polynomial, be those moment functionals defined by

$$
\left\langle\sigma^{\prime}, p(x)\right\rangle=-\left\langle\sigma, p^{\prime}(x)\right\rangle \quad(p(x) \in \mathscr{P})
$$

and

$$
\langle\pi(x) \sigma, p(x)\rangle=\langle\sigma, \pi(x) p(x)\rangle \quad(p(x) \in \mathscr{P}) .
$$

It is easy then to obtain the following Leibniz rule for any moment functional $\sigma$ and polynomial $\pi(x)$ as

$$
(\pi(x) \sigma)^{\prime}=\pi^{\prime}(x) \sigma+\pi(x) \sigma^{\prime} .
$$

Lemma 2.2. Let $\sigma$ be a moment functional and $\pi(x)$ a polynomial.

(i) Then $\sigma=0$ if and only if $\sigma^{\prime}=0$.

(ii) If $\sigma$ is quasi-definite and $\pi(x) \sigma=0$, then $\pi(x) \equiv 0$. 
Proof. (i) If $\sigma=0$, then $\left\langle\sigma^{\prime}, x^{n}\right\rangle=-n\left\langle\sigma, x^{n-1}\right\rangle=0$ for any integer $n \geq 0$ so that $\sigma^{\prime}=0$. Conversely, if $\sigma^{\prime}=0$, then $\left\langle\sigma, x^{n}\right\rangle=\left\langle\sigma, \frac{1}{n+1}\left(x^{n+1}\right)^{\prime}\right\rangle=$ $\frac{-1}{n+1}\left\langle\sigma^{\prime}, x^{n+1}\right\rangle=0$ for any integer $n \geq 0$ so that $\sigma=0$.

(ii) Assume that $\sigma$ is quasi-definite and $\pi(x) \sigma=0$. Let $\left\{P_{n}(x)\right\}_{n=0}^{\infty}$ be an OPS relative to $\sigma$, satisfying the orthogonality condition $\left\langle\sigma, P_{m}(x) P_{n}(x)\right\rangle=$ $K_{n} \delta_{m n}(m$ and $n \geq 0)$ with $K_{n} \neq 0$. Suppose $\operatorname{deg}(\pi)=n \geq 0$ and write $\pi(x)=\sum_{k=0}^{n} C_{k} P_{k}(x)$, where $C_{n} \neq 0$. Then we have

$$
0=\left\langle\pi(x) \sigma, P_{n}(x)\right\rangle=\sum_{k=0}^{n} C_{k}\left\langle\sigma, P_{k}(x) P_{n}(x)\right\rangle=C_{n} K_{n},
$$

so that $C_{n}=0$, which is a contradiction. Hence $\pi(x) \equiv 0$.

\section{MAIN THEOREM}

For a linear differential operator

$$
L[\cdot]=\sum_{i=0}^{N} a_{i}(x) D^{i}
$$

where $D=d / d x$, each $a_{i}(x)$ is a real-valued function in $C^{i}(I)$, and $I$ is an open interval in $\mathbb{R}$, we let $L^{*}[\cdot]$ be the formal adjoint of $L[\cdot]$ defined by

$$
L^{*}[y]=\sum_{i=0}^{N}(-1)^{i}\left(a_{i} y\right)^{(i)}
$$

Proposition 3.1. For a differential operator $L_{N}[\cdot]$ in (1.1) and a Sobolev inner product $\phi(\cdot, \cdot)$ in $(2.7)$, the following are equivalent.

(a) $L_{N}[\cdot]$ is symmetric on polynomials relative to $\phi(\cdot, \cdot)$, that is,

$$
\phi\left(L_{N}[p], q\right)=\phi\left(p, L_{N}[q]\right)
$$

for any polynomials $p(x)$ and $q(x)$.

(b) Moment functionals $\sigma$ and $\tau$ satisfy

$$
L_{N}[q] \sigma-L_{N}^{*}[q \sigma]=\left(L_{N}[q]^{\prime} \tau\right)^{\prime}-L_{N}^{*}\left[\left(q^{\prime} \tau\right)^{\prime}\right]
$$

for any polynomial $q(x)$.

Proof. For any polynomials $p(x)$ and $q(x)$, we have

$$
\phi\left(L_{N}[p], q\right)=\left\langle\sigma, L_{N}[p] q\right\rangle+\left\langle\tau, L_{N}[p]^{\prime} q^{\prime}\right\rangle=\left\langle L_{N}^{*}[q \sigma], p\right\rangle-\left\langle L_{N}^{*}\left[\left(q^{\prime} \tau\right)^{\prime}\right], p\right\rangle
$$

and

$$
\phi\left(p, L_{N}[q]\right)=\left\langle\sigma, p L_{N}[q]\right\rangle+\left\langle\tau, p^{\prime} L_{N}[q]^{\prime}\right\rangle=\left\langle L_{N}[q] \sigma, p\right\rangle-\left\langle\left(L_{N}[q]^{\prime} \tau\right)^{\prime}, p\right\rangle .
$$

Since $p(x)$ can be any polynomial, the statements $(\mathrm{a})$ and $(\mathrm{b})$ are equivalent.

With the notation $\sigma \pi(x):=\pi(x) \sigma$ for any polynomial $\pi(x)$ and any moment functional $\sigma$, we may view the equation (3.2) as a differential equation of order $N+2$ in $q(x)$. To be precise, we may rewrite the equation (3.2) as

$$
\sum_{k=0}^{N+2} R_{k+1}(\sigma, \tau) q^{(k)}(x)=0
$$


where

$$
\begin{aligned}
R_{N+3}(\sigma, \tau)=R_{N+3}(\tau)=\left[(-1)^{N}-1\right] \ell_{N} \tau & \\
R_{N+2}(\sigma, \tau)= & R_{N+2}(\tau)=\left[(-1)^{N}(N+1)-1\right]\left(\ell_{N} \tau\right)^{\prime}-\left[(-1)^{N}+1\right] \\
& \times\left(\ell_{N}^{\prime}+\ell_{N-1}\right) \tau ; \\
R_{k+1}(\sigma, \tau)= & \sum_{j=0}^{N-k+2}(-1)^{j+k}\left(\begin{array}{c}
j+k-2 \\
k-2
\end{array}\right)\left(\ell_{j+k-2} \tau\right)^{(j)}-\ell_{k-2} \tau \\
& -2\left(\ell_{k-1} \tau\right)^{\prime}-\left(\ell_{k} \tau\right)^{\prime \prime} \\
& -\sum_{j=0}^{N-k+1}(-1)^{j+k}\left(\begin{array}{c}
j+k-1 \\
k-1
\end{array}\right)\left(\ell_{j+k-1} \tau^{\prime}\right)^{(j)}+\ell_{k-1} \tau^{\prime} \\
& +\left(\ell_{k} \tau^{\prime}\right)^{\prime}-\sum_{j=0}^{N-k}(-1)^{j+k}\left(\begin{array}{c}
j+k \\
k
\end{array}\right)\left(\ell_{j+k} \sigma\right)^{(j)} \\
& +\ell_{k} \sigma, \quad k=0,1, \cdots, N,
\end{aligned}
$$

where $\left(\begin{array}{l}n \\ k\end{array}\right)=0$ for $k<0$ and $\ell_{k}(x)=0$ for $k \leq 0$.

Hence, the condition (b) in Proposition 3.1 is also equivalent to the condition that $\sigma$ and $\tau$ satisfy $N+3$ functional equations:

$$
R_{k+1}(\sigma, \tau)=0, \quad k=0,1, \cdots, N+2 .
$$

Are these equations independent? No. For example, if $N$ is even, then $R_{N+3}(\tau) \equiv 0$ and if $N$ is odd, then $R_{N+3}(\tau)=-2 \ell_{N} \tau$ and $R_{N+2}(\tau)=$ $-(N+2)\left(\ell_{N} \tau\right)^{\prime}$ so that $R_{N+3}(\tau)=0$ if and only if $R_{N+2}(\tau)=0$ (cf. Lemma 2.2). In fact, the above $N+3$ equations can be reduced to $\left[\frac{N+3}{2}\right]$ equivalent equations as we shall see below.

We set

$$
S_{k+1}(\sigma)=\sum_{j=0}^{N-k}(-1)^{j+k}\left(\begin{array}{c}
j+k \\
k
\end{array}\right)\left(\ell_{j+k} \sigma\right)^{(j)}-\left(\ell_{k} \sigma\right)
$$

$$
T_{k+1}(\sigma)=\sum_{j=0}^{N-k+1}(-1)^{j+k}\left(\begin{array}{c}
j+k-1 \\
k-1
\end{array}\right)\left(\ell_{j+k-1} \sigma\right)^{(j)}-\ell_{k-1} \sigma-\left(\ell_{k} \sigma\right)^{\prime}
$$

$$
\begin{gathered}
U_{k+1}(\sigma)=\sum_{j=0}^{N-k+2}(-1)^{j+k}\left(\begin{array}{c}
j+k-2 \\
k-2
\end{array}\right)\left(\ell_{j+k-2} \sigma\right)^{(j)}-\ell_{k-2} \sigma \\
-2\left(\ell_{k-1} \sigma\right)^{\prime}-\left(\ell_{k} \sigma\right)^{\prime \prime},
\end{gathered}
$$

$k=0,1, \cdots, N$. Then

$$
R_{k+1}(\sigma, \tau)=U_{k+1}(\tau)-T_{k+1}\left(\tau^{\prime}\right)-S_{k+1}(\sigma), \quad k=0,1, \cdots, N .
$$

Proposition 3.2. The statement (a) or (b) in Proposition 3.1 is also equivalent to any one of the following.

(c) $\sigma$ and $\tau$ satisfy $N+3$ functional equations in (3.7). 
(d) $\sigma$ and $\tau$ satisfy $r+1$ functional equations

$$
R_{2 k+2}(\sigma, \tau)=0, \quad k=0,1, \cdots, r:=\left[\frac{N+1}{2}\right] .
$$

(e) $\sigma$ and $\tau$ satisfy $r+1$ functional equations

$$
R_{2 k+1}(\sigma, \tau)=0, \quad k=0,1, \cdots, r:=\left[\frac{N+1}{2}\right] .
$$

We first need several lemmas. For $j=0,1, \cdots$, we set

$$
c_{j}=\frac{2\left(2^{2 j+2}-1\right) B_{2 j+2}}{(2 j+2) !},
$$

where $\left\{B_{2 j}\right\}_{j=1}^{\infty}$ are the Bernoulli numbers defined by

$$
\frac{x}{e^{x}-1}=1-\frac{1}{2} x+\sum_{j=1}^{\infty} B_{2 j} x^{2 j}
$$

Lemma 3.3. The following combinatorial indentities hold:

$$
\begin{aligned}
& \frac{1}{(2 i+1) !}=\sum_{j=0}^{i} \frac{c_{j}}{(2 i-2 j) !}+c_{i} \quad(i=0,1, \cdots) ; \\
& \frac{1}{(2 i+2) !}=\sum_{j=0}^{i} \frac{c_{j}}{(2 i-2 j+1) !} \quad(i=0,1, \cdots) .
\end{aligned}
$$

Proof. See Lemma 4.4 in [11].

Using Lemma 3.3, Littlejohn and Race [11] proved

Lemma 3.4. If $N=2 r$ is even, then for any moment functional $\sigma$, we have

$$
S_{N-2 k-1}(\sigma)-\sum_{j=0}^{k} c_{j} P(N-2 k+2 j-1,2 j+1) S_{N-2 k+2 j}^{(2 j+1)}(\sigma)=0,
$$

$k=0,1, \cdots, r-1$, where $P(n, k)=n(n-1) \cdots(n-k+1)$.

Proof. See Lemma 4.5 in [11].

Lemma 3.5. For any non-negative integers $n$ and $i$, we have the following combinatorial identities: 


$$
\begin{aligned}
& \left(\begin{array}{c}
n+2 i+3 \\
n
\end{array}\right)-\sum_{j=0}^{i} c_{j} P(n+2 j+3,2 j+1)\left(\begin{array}{l}
n+2 i+3 \\
n+2 j+1
\end{array}\right) \\
& -2 c_{i+1} P(n+2 i+5,2 i+3)-c_{i} P(n+2 i+3,2 i+1)=0 ;
\end{aligned}
$$

$$
\begin{gathered}
\left(\begin{array}{c}
n+2 i+4 \\
n
\end{array}\right)-\sum_{j=0}^{i} c_{j} P(n+2 j+3,2 j+1)\left(\begin{array}{c}
n+2 i+4 \\
n+2 j+1
\end{array}\right) \\
-c_{i+1}(n+2 i+2) P(n+2 i+5,2 i+3)=0
\end{gathered}
$$

$$
\begin{gathered}
\left(\begin{array}{c}
n+2 i+3 \\
n
\end{array}\right)-\sum_{j=0}^{i} c_{j} P(n+2 j+2,2 j+1)\left(\begin{array}{l}
n+2 i+3 \\
n+2 j+1
\end{array}\right) \\
-2 c_{i+1} P(n+2 i+4,2 i+3)=0 \\
\left(\begin{array}{c}
n+2 i+2 \\
n
\end{array}\right)--\sum_{j=0}^{i} c_{j} P(n+2 j+2,2 j+1)\left(\begin{array}{l}
n+2 i+2 \\
n+2 j+1
\end{array}\right) \\
+c_{i} P(n-2 i+2,2 i+1)=0 .
\end{gathered}
$$

Proof. The identities (3.18) and (3.19) follow immediately from (3.15), (3.16), and

$$
\left(\begin{array}{c}
n+2 \\
k
\end{array}\right)=\left(\begin{array}{l}
n+4 \\
k+2
\end{array}\right)-2\left(\begin{array}{l}
n+3 \\
k+2
\end{array}\right)+\left(\begin{array}{l}
n+2 \\
k+2
\end{array}\right)
$$

The identities (3.20) and (3.21) follow immediately from (3.15), (3.16), and

$$
\left(\begin{array}{l}
n+1 \\
k+1
\end{array}\right)=\left(\begin{array}{c}
n \\
k+1
\end{array}\right)+\left(\begin{array}{l}
n \\
k
\end{array}\right)
$$

Lemma 3.6. If $N=2 r$ is even, then for any moment functional $\sigma$, we have (3.22)

$$
\begin{aligned}
& U_{N-2 k-1}(\sigma)-\sum_{j=0}^{k} c_{j} P(N-2 k+2 j-1,2 j+1) U_{N-2 k+2 j}^{(2 j+1)}(\sigma) \\
& =c_{k+1} P(N+1,2 k+3)\left[(N-2)\left(\ell_{N} \sigma\right)^{(2 k+4)}-2\left(\ell_{N-1} \tau\right)^{(2 k+3)}\right],
\end{aligned}
$$

$$
\begin{gathered}
T_{N-2 k-1}(\sigma)-\sum_{j=0}^{k} c_{j} P(N-2 k+2 j-1,2 j+1)^{e} T_{N-2 k+2 j}^{(2 j+1)}(\sigma) \\
=-2 c_{k+1} P(N+1,2 k+3)\left(\ell_{N} \sigma\right)^{(2 k+3)}
\end{gathered}
$$

$k=0,1, \cdots, r-1$.

Proof. For simplicity of notation, we let $c_{j} P(N-2 k+2 j-1,2 j+1)=c_{j}(m)$ and $N-2 k=m$. Then 


$$
\begin{aligned}
& U_{m-1}(\sigma)-\sum_{j=0}^{k} c_{j}(m) U_{m+2 j}^{(2 j+1)}(\sigma) \\
& =\sum_{j=0}^{2 k+4}(-1)^{j}\left(\begin{array}{c}
m+j-4 \\
m-4
\end{array}\right)\left(\ell_{m+j-4} \sigma\right)^{(j)}-\ell_{m-4} \sigma-2\left(\ell_{m-3} \sigma\right)^{\prime}-\left(\ell_{m-2} \sigma\right)^{\prime \prime} \\
& -\sum_{j=0}^{k} c_{j}(m)\left\{\sum_{i=0}^{2 k-2 j+3}(-1)^{i+1}\left(\begin{array}{c}
m+2 j+i-3 \\
m+2 j-3
\end{array}\right)\left(\ell_{m+2 j+i-3} \sigma\right)^{(i+2 j+1)}\right. \\
& \left.-\left(\ell_{m+2 j-3} \sigma\right)^{(2 j+1)}-2\left(\ell_{m+2 j-2} \sigma\right)^{(2 j+2)}-\left(\ell_{m+2 j-1} \sigma\right)^{(2 j+3)}\right\} \\
& =-\sum_{j=-1}^{k}\left(\begin{array}{c}
m+2 j-1 \\
m-4
\end{array}\right)\left(\ell_{m+2 j-1} \sigma\right)^{(2 j+3)}-2\left(\ell_{m-3} \sigma\right)^{\prime}+\sum_{i=0}^{k} \sum_{j=0}^{i} c_{j}(m) \\
& \times\left(\begin{array}{l}
m+2 i-1 \\
m+2 j-3
\end{array}\right)\left(\ell_{m+2 i-1} \sigma\right)^{(2 i+3)}+2 \sum_{j=-1}^{k-1} c_{j+1}(m)\left(\ell_{m+2 j-1} \sigma\right)^{(2 j+3)} \\
& +\sum_{j=0}^{k} c_{j}(m)\left(\ell_{m+2 j-1} \sigma\right)^{(2 j+3)}+\sum_{j=-1}^{k}\left(\begin{array}{c}
m+2 j \\
m-4
\end{array}\right)\left(\ell_{m+2 j} \sigma\right)^{(2 j+4)} \\
& -\left(\ell_{m-2} \sigma\right)^{\prime \prime}-\sum_{i=0}^{k} \sum_{j=0}^{i} c_{j}(m)\left(\begin{array}{c}
m+2 i \\
m+2 j-3
\end{array}\right)\left(\ell_{m+2 i} \sigma\right)^{(2 i+4)}-\sum_{j=0}^{k} c_{j}(m) \\
& \times(m+2 j-2)\left(\ell_{m+2 j-2} \sigma\right)^{(2 j+2)}+2 \sum_{j=-1}^{k-1} c_{j+1}(m)\left(\ell_{m+2 j} \sigma\right)^{(2 j+4)} \\
& =\sum_{i=0}^{k}\left\{-\left(\begin{array}{c}
m+2 i-1 \\
m-4
\end{array}\right)+\sum_{j=0}^{i} c_{j}(m)\left(\begin{array}{c}
m+2 i-1 \\
m+2 j-3
\end{array}\right)+2 c_{i+1}(m)+c_{i}(m)\right\} \\
& \times\left(\ell_{m+2 i-1} \sigma\right)^{(2 i+3)} \\
& +\sum_{i=0}^{k}\left\{\left(\begin{array}{c}
m+2 i \\
m-4
\end{array}\right)-\sum_{j=0}^{i} c_{j}(m)\left(\begin{array}{c}
m+2 i \\
m+2 j-3
\end{array}\right)-c_{i+1}(m)(m+2 i-2)\right\} \\
& \times\left(\ell_{m+2 i} \sigma\right)^{(2 i+4)}+c_{k+1}(m)\left[(N-2)\left(\ell_{N} \sigma\right)^{(2 k+4)}-2\left(\ell_{N-1} \sigma\right)^{(2 k+3)}\right] \\
& =c_{k+1}(m)\left[(N-2)\left(\ell_{N} \sigma\right)^{(2 k+4)}-2\left(\ell_{N-1} \sigma\right)^{(2 k+3)}\right]
\end{aligned}
$$

since the coefficients in the first and the second summations vanish by (3.18) and (3.19) respectively. Similarly we have

$$
\begin{aligned}
T_{m-1}(\sigma)- & \sum_{j=0}^{k} c_{j}(m) T_{m+2 j}^{(2 j+1)}(\sigma) \\
= & \sum_{i=0}^{k}\left\{-\left(\begin{array}{c}
m+2 i \\
m-3
\end{array}\right)+\sum_{j=0}^{i} c_{j}(m)\left(\begin{array}{c}
m+2 i \\
m+2 j-2
\end{array}\right)+2 c_{i+1}(m)\right\} \\
& \times\left(\ell_{m+2 i} \sigma\right)^{(2 i+3)}
\end{aligned}
$$




$$
\begin{aligned}
& +\sum_{i=0}^{k}\left\{\left(\begin{array}{c}
m+2 i-1 \\
m-3
\end{array}\right)-\sum_{j=0}^{i} c_{j}(m)\left(\begin{array}{l}
m+2 i-1 \\
m+2 j-2
\end{array}\right)+c_{i}(m)\right\} \\
& \times\left(\ell_{m+2 i-1} \sigma\right)^{(2 i+2)}-2 c_{k+1}(m)\left(\ell_{N} \sigma\right)^{(2 k+3)} \\
& =-2 c_{k+1}(m)\left(\ell_{N} \sigma\right)^{(2 k+3)}
\end{aligned}
$$

since the coefficients in the first and the second summations vanish by (3.20) and (3.21) respectively.

Proof of Proposition 3.2. We only need to show the equivalence of the statements (c), (d), and (e). It is trivial that (c) implies (d) and (e).

$(d) \Rightarrow(c)$ : We first claim that

$$
R_{2 k-1}(\sigma, \tau)=\sum_{j=0}^{r-k+1} c_{j} P(2 k+2 j-1,2 j+1) R_{2 k+2 j}^{(2 j+1)}(\sigma, \tau),
$$

$k=1,2, \cdots, r+1$, where $r:=\left[\frac{N+1}{2}\right]$.

First we assume $N=2 r$ is even. Since $S_{N+1}(\sigma)=0$, we have from (3.5) and (3.6) with $k=N$

$$
\begin{aligned}
R_{N+1}(\sigma, \tau) & =U_{N+1}(\tau)-T_{N+1}\left(\tau^{\prime}\right) \\
& =\frac{N(N+1)}{2}\left(\ell_{N} \tau\right)^{\prime \prime}-(N+1)\left[\left(\ell_{N-1} \tau\right)^{\prime}+\left(\ell_{N}^{\prime} \tau\right)^{\prime}\right] \\
& =\frac{N+1}{2} R_{N+2}^{\prime}(\tau)
\end{aligned}
$$

so that (3.24) holds for $k=r+1$ since $c_{0}=\frac{1}{2}$.

For $1 \leq k \leq r$, we have from (3.17), (3.22), and (3.23)

$$
\begin{aligned}
& R_{2 k-1}(\sigma, \tau)-\sum_{j=0}^{r-k+1} c_{j} P(2 k+2 j-1,2 j+1) R_{2 k+2 j}^{(2 j+1)}(\sigma, \tau) \\
&=\left(U_{2 k-1}(\tau)-T_{2 k-1}\left(\tau^{\prime}\right)-S_{2 k-1}(\sigma)\right)-\sum_{j=0}^{r-k+1} c_{j} P(2 k+2 j-1,2 j+1) \\
& \quad \times\left(U_{2 k+2 j}^{(2 j+1)}(\tau)-T_{2 k+2 j}^{(2 j+1)}\left(\tau^{\prime}\right)-S_{2 k+2 j}^{(2 j+1)}(\sigma)\right) \\
&=c_{r-k+1} P(N+1, N-2 k+3) \\
& \quad \times\left[(N-2)\left(\ell_{N} \tau\right)^{(N-2 k+4)}-2\left(\ell_{N-1} \tau\right)^{(N-2 k+3)}\right] \\
& \quad+2 c_{r-k+1} P(N+1, N-2 k+3)\left(\ell_{N} \tau^{\prime}\right)^{(N-2 k+3)} \\
& \quad-c_{r-k+1} P(N+1, N-2 k+3) R_{N+2}^{(N-2 k+3)}(\tau) \\
&= 0
\end{aligned}
$$

so that (3.24) holds also for $1 \leq k \leq r$.

For $N=2 r-1$ odd, we let $L_{N+1}[\cdot]:=\ell_{N+1}(x) D^{N+1}+L_{N}[\cdot]$ with $\ell_{N+1}(x) \equiv 0$ and let $\left\{\widetilde{R}_{k}(\sigma, \tau)\right\}_{k=1}^{N+4}$ be the coefficients in (3.3) corresponding to $L_{N+1}[\cdot]$. Then

$$
\widetilde{R}_{N+4}(\sigma, \tau) \equiv 0 \quad \text { and } \quad \widetilde{R}_{k}(\sigma, \tau)=R_{k}(\sigma, \tau), \quad 1 \leq k \leq N+3
$$


Hence, in the case, we also have (3.24). Now (3.24) implies that if $R_{2 k}(\sigma, \tau)=$ $0,1 \leq k \leq r+1$, then $R_{k}(\sigma, \tau)=0,1 \leq k \leq 2 r+2$. Hence (d) implies (c) since $R_{N+3}(\tau)=R_{2 r+3}(\tau) \equiv 0$ when $N=2 r$ is even.

(e) $\Rightarrow$ (c): We may solve $r+1$ equations in (3.24) successively for $R_{2 r+2}^{\prime}, R_{2 r}^{\prime}$, $\cdots, R_{2}^{\prime}$ as

$$
R_{2 k}^{\prime}(\sigma, \tau)=\sum_{j=0}^{r-k+1} \gamma_{j k} R_{2 k+2 j-1}^{(2 j)},
$$

where $\gamma_{0 k}=\left(c_{0}(2 k-1)\right)^{-1}=\frac{2}{2 k-1}, 1 \leq k \leq r+1$. Since for any $k=$ $1, \cdots, N+3, R_{k}^{\prime}(\sigma, \tau)=0$ if and only if $R_{k}(\sigma, \tau)=0$ by Lemma 2.2, (3.25) implies that if $R_{2 k+1}(\sigma, \tau)=0,0 \leq k \leq r$, then $R_{k}(\sigma, \tau)=0,1 \leq k \leq 2 r+2$. Hence, (e) implies (c) since $R_{N+3}(\tau)=R_{2 r+3}(\tau) \equiv 0$ when $N=2 r$ is even.

Remark 3.1. In fact, Lemma 3.4 and Lemma 3.6 mean that differential operators on both sides of the equations (3.17), (3.22), and (3.23) are the same respectively.

Remark 3.2. It can be easily shown that the set of $r+1$ equations in (3.12) or (3.13) is also equivalent to the set of $r+1$ equations consisting of one equation from each pair $R_{2 k-1}(\sigma, \tau)=0$ and $R_{2 k}(\sigma, \tau)=0$ for $k=1,2, \cdots, r+1$.

We are now ready to give our main result.

Theorem 3.7. Let $\left\{P_{n}(x)\right\}_{n=0}^{\infty}$ be an SOPS relative to a quasi-definite Sobolev inner product $\phi(\cdot, \cdot \cdot)$ in (2.7). Then any one of the statements (a), (b), (c), (d), and (e) in Proposition 3.1 and in Proposition 3.2 is also equivalent to the following.

(f) For each $n \geq 0, P_{n}(x)$ satisfies the differential equation (1.1).

Moreover, if any one of these equivalent conditions holds, then $N=2 r$ must be even.

Proof. By Proposition 3.1 and Proposition 3.2, it suffices to show that the statement (a) in Proposition 3.1 and the above statement (f) are equivalent.

(a) $\Rightarrow$ (f): For any fixed integer $n \geq 1, L_{N}\left[P_{n}\right](x)$ is a polynomial of degree $\leq n$ so that we may write $L_{N}\left[P_{n}\right](x)$ as

$$
L_{N}\left[P_{n}\right](x)=\sum_{j=0}^{n} C_{j} P_{j}(x)
$$

for some constants $C_{j}, j=0,1, \cdots, n$. Then for any integer $k$ with $0 \leq$ $k<n$,

$$
\begin{aligned}
C_{k} \phi\left(P_{k}(x), P_{k}(x)\right) & =\phi\left(\sum_{j=0}^{n} C_{j} P_{j}(x), P_{k}(x)\right) \\
& =\phi\left(L_{N}\left[P_{n}\right](x), P_{k}(x)\right)=\phi\left(P_{n}(x), L_{N}\left[P_{k}\right](x)\right)=0
\end{aligned}
$$

since $L_{N}\left[P_{k}\right](x)$ is a polynomial of degree $\leq k$ and $k<n$. Hence, $C_{k}=$ $0, k=0,1, \cdots, n-1$, and $L_{N}\left[P_{n}\right](x)=C_{n} P_{n}(x)=\lambda_{n} P_{n}(x)$ by comparing the coefficients of $x^{n}$ from both sides. 
(f) $\Rightarrow$ (a): From the orthogonality of $\left\{P_{n}(x)\right\}_{n=0}^{\infty}$, we have

$$
\begin{aligned}
0 & =\left(\lambda_{m}-\lambda_{n}\right) \phi\left(P_{m}(x), P_{n}(x)\right)=\phi\left(\lambda_{m} P_{m}(x), P_{n}(x)\right)-\phi\left(P_{m}(x), \lambda_{n} P_{n}(x)\right) \\
& =\phi\left(L_{N}\left[P_{m}\right](x), P_{n}(x)\right)-\phi\left(P_{m}(x), L_{N}\left[P_{n}\right](x)\right)
\end{aligned}
$$

for every $m$ and $n \geq 0$. Since $\left\{P_{n}(x)\right\}_{n=0}^{\infty}$ is a basis of $\mathscr{P}$, we have (3.1).

Finally assume that $N$ is odd and $\ell_{N}(x) \not \equiv 0$. We know that $\sigma$ and $\tau$ satisfy the condition (3.12). In particular,

$$
R_{N+3}(\tau)=-2 \ell_{N} \tau=0
$$

and

$$
\begin{aligned}
R_{N+1}(\sigma, \tau)= & \frac{N(1-N)-2}{2}\left(\ell_{N} \tau\right)^{\prime \prime}+(N-3)\left(\ell_{N-1} \tau\right)^{\prime} \\
& -(N-1)\left(\ell_{N} \tau^{\prime}\right)^{\prime}+2 \ell_{N-1} \tau^{\prime}-2 \ell_{N-2} \tau+2 \ell_{N} \sigma=0 .
\end{aligned}
$$

Equivalently, $\sigma$ and $\tau$ satisfy

$$
\ell_{N} \tau=0,
$$

$$
\begin{aligned}
& (N-1)\left(\ell_{N}^{\prime}+\ell_{N-1}\right) \tau^{\prime} \\
& \quad+\left[(N-1) \ell_{N}^{\prime \prime}+(N-3) \ell_{N-1}^{\prime}-2 \ell_{N-2}\right] \tau+2 \ell_{N} \sigma=0 .
\end{aligned}
$$

Hence, we have $\ell_{N}(x) \sigma=\alpha(x) \tau^{\prime}+\beta(x) \tau$ for some polynomial $\alpha(x)$ and $\beta(x)$. Then for any polynomial $\pi(x)$, we have from (3.26)

$$
\begin{aligned}
\phi\left(\pi, \ell_{N}^{3}\right) & =\left\langle\sigma, \pi \ell_{N}^{3}\right\rangle+\left\langle\tau, 3 \pi^{\prime} \ell_{N}^{2} \ell_{N}^{\prime}\right\rangle \\
& =\left\langle\alpha \tau^{\prime}+\beta \tau, \pi \ell_{N}^{2}\right\rangle+\left\langle\ell_{N} \tau, 3 \pi^{\prime} \ell_{N} \ell_{N}^{\prime}\right\rangle \\
& =\left\langle\ell_{N} \tau, \beta \pi \ell_{N}-(\alpha \pi)^{\prime} \ell_{N}-2 \alpha \pi \ell_{N}^{\prime}\right\rangle \\
& =0 .
\end{aligned}
$$

It contradicts the fact that $\phi\left(P_{n}(x), \ell_{N}^{3}(x)\right) \neq 0$ when $n=\operatorname{deg}\left(\ell_{N}^{3}\right)$.

We call the $r+1$ functional equations in (3.12) or (3.13) the moment equations of $L_{N}[\cdot]$ (relative to $\left.\phi(\cdot, \cdot)\right)$. 3.7:

When $\tau \equiv 0$, we may obtain Krall's theorem [6] as a special case of Theorem

Corollary 3.8. Let $\sigma$ be a quasi-definite moment functional and $\left\{P_{n}(x)\right\}_{n=0}^{\infty}$ an OPS relative to $\sigma$. Then, $\left\{P_{n}(x)\right\}_{n=0}^{\infty}$ satisfy the differential equation (1.1) if and only if $\sigma$ satisfies $r:=\left[\frac{N+1}{2}\right]$ functional equations

$$
S_{2 k+2}(\sigma)=0, \quad k=0,1, \cdots, r-1 .
$$

Moreover, in this case, $N=2 r$ must be even.

Proof. By Theorem 3.7, $\left\{P_{n}(x)\right\}_{n=0}^{\infty}$ satisfy the differential equation (1.1) if and only if $R_{2 k+2}(\sigma, 0)=0, k=0,1, \cdots, r$. However, $R_{2 k+2}(\sigma, 0)=$ $-S_{2 k+2}(\sigma), k=0,1, \cdots, r-1$, and $R_{2 r+2}(\sigma, 0) \equiv 0$. Hence, the conclusion follows.

Remark 3.3. In [11], Littlejohn and Race showed that the set of $r$ equations in (3.28) is also equivalent to

$$
W_{k+1}(\sigma)=\sum_{i=2 k+1}^{N}(-1)^{i}\left(\begin{array}{c}
i-k-1 \\
k
\end{array}\right)\left(\ell_{i} \sigma\right)^{(i-2 k-1)}=0, \quad k=0,1, \cdots, r-1 .
$$


If we rewrite (3.29) in terms of moments $\left\{\sigma_{n}\right\}_{n=0}^{\infty}$ of $\sigma$, then we obtain

$$
S_{k}(m)=\sum_{i=2 k+1}^{N} \sum_{j=0}^{i}\left(\begin{array}{c}
i-k-1 \\
k
\end{array}\right) P(m-2 k-1, i-2 k-1) \ell_{i, i-j} \sigma_{m-j}=0
$$

for $k=0,1, \cdots, r-1$ and $m=2 k+1,2 k+2, \cdots$. The moment equations in (3.30) were found and used by H. L. Krall [6] in his characterization of differential equations having OPS's of solutions.

Remark 3.4. If we view the equations in (3.28) or in (3.29) as true differential equations for functions, then any common non-trivial classical solution $s(x)$ (if it exists) is a symmetry factor of the differential operator $L_{N}[\cdot]$, that is, $s(x) L_{N}[\cdot]$ is formally symmetric (see [11] for details).

Finally, we give several examples illustrating our main theorem.

Example 3.1. We consider the differential equation (1.1) with $N=2$ :

$$
L_{2}[y](x)=\ell_{2}(x) y^{\prime \prime}(x)+\ell_{1}(x) y^{\prime}(x)=\left[n(n-1) \ell_{22}+n \ell_{11}\right] y(x) .
$$

In this case, there are four moment equations:

$$
\begin{gathered}
R_{4}(\tau)=2\left(\ell_{2} \tau\right)^{\prime}-2\left(\ell_{2}^{\prime}+\ell_{1}\right) \tau=0 \\
R_{3}(\tau)=-3\left(\ell_{1} \tau\right)^{\prime}+3\left(\ell_{2} \tau^{\prime}\right)^{\prime}=\frac{3}{2} R_{4}^{\prime}(\tau)=0 \\
R_{2}(\sigma, \tau)=-\left(\ell_{1} \tau\right)^{\prime \prime}+\left(\ell_{2} \tau^{\prime}\right)^{\prime \prime}-2\left(\ell_{2} \sigma\right)^{\prime}+2 \ell_{1} \sigma=0 \\
R_{1}(\sigma, \tau)=-\left(\ell_{2} \sigma\right)^{\prime \prime}+\left(\ell_{1} \sigma\right)^{\prime}=\frac{1}{2} R_{2}^{\prime}(\sigma, \tau)-\frac{1}{4} R_{4}^{(3)}(\tau)=0
\end{gathered}
$$

which can be simplified into the following equivalent set of two uncoupled equations

$$
\left(\ell_{2} \sigma\right)^{\prime}-\ell_{1} \sigma=0, \quad \ell_{2} \tau^{\prime}-\ell_{1} \tau=0 .
$$

It is shown in [9] that there are essentially (i.e., up to a real linear change of variable) eleven distinct SOPS's that satisfy the differential equation (3.31). In particular, if $\sigma$ is quasi-definite, then we obtain only classical OP's and an SOPS, which is not an OPS, can arise only when $\sigma$ is not quasi-definite (in fact, discrete) and $\tau$ is quasi-definite (see [9] for details). For example, the Laguerre polynomials $\left\{L_{n}^{(-1)}(x)\right\}_{n=0}^{\infty}$ satisfy

$$
x y^{\prime \prime}(x)-x y^{\prime}(x)=-n y(x)
$$

and is orthogonal relative to

$$
\phi(p, q)=A p(0) q(0)+\int_{0}^{\infty} p^{\prime}(x) q^{\prime}(x) e^{-x} d x
$$

where $A \neq 0$.

Example 3.2. We consider the differential equation (1.1) with $N=4$ :

$$
L_{4}[y](x)=\ell_{4}(x) y^{(4)}(x)+\ell_{3}(x) y^{(3)}(x)+\ell_{2}(x) y^{\prime \prime}(x)+\ell_{1}(x) y^{\prime}(x)=\lambda_{n} y(x) .
$$


In this case, there are six moment equations:

$$
\begin{aligned}
R_{6}(\sigma, \tau)= & R_{6}(\tau)=4\left(\ell_{4} \tau\right)^{\prime}-2\left(\ell_{4}^{\prime}+\ell_{3}\right) \tau=0, \\
R_{5}(\sigma, \tau)= & R_{5}(\tau)=\frac{5}{2} R_{6}^{\prime}(\tau)=0, \\
R_{4}(\sigma, \tau)= & 4\left(\ell_{4} \tau\right)^{(3)}-3\left(\ell_{3} \tau\right)^{\prime \prime}+2\left(\ell_{2} \tau\right)^{\prime}-\ell_{1} \tau+6\left(\ell_{4} \tau^{\prime}\right)^{\prime \prime}-3\left(\ell_{3} \tau^{\prime}\right)^{\prime}+\ell_{2} \tau^{\prime} \\
& -\left(\ell_{1}+2 \ell_{2}^{\prime}+\ell_{3}^{\prime \prime}\right) \tau-\left(\ell_{2}+\ell_{3}^{\prime}\right) \tau^{\prime}+2 \ell_{3} \sigma-4\left(\ell_{4} \sigma\right)^{\prime}=0, \\
R_{3}(\sigma, \tau)= & \frac{3}{2} R_{4}^{\prime}(\sigma, \tau)-\frac{5}{2} R_{6}^{(3)}(\tau)=0, \\
R_{2}(\sigma, \tau)= & \left(\ell_{4} \tau^{\prime}\right)^{(4)}-\left(\ell_{3} \tau^{\prime}\right)^{(3)}+\left(\ell_{2} \tau^{\prime}\right)^{\prime \prime}-\left(\ell_{1} \tau^{\prime}\right)^{\prime}-\ell_{1}^{\prime} \tau^{\prime} \\
& -4\left(\ell_{4} \sigma\right)^{(3)}+3\left(\ell_{3} \sigma\right)^{\prime \prime}-2\left(\ell_{2} \sigma\right)^{\prime}+2 \ell_{1} \sigma=0, \\
R_{1}(\sigma, \tau)= & \frac{1}{2} R_{2}^{\prime}(\sigma, \tau)-\frac{1}{4} R_{4}^{(3)}(\sigma, \tau)+\frac{1}{8} R_{6}^{(5)}(\tau)=0 .
\end{aligned}
$$

We may replace the three moment equations $R_{2 k}(\sigma, \tau)=0, k=1,2,3$, by

$$
\begin{gathered}
2\left(\ell_{4} \tau\right)^{\prime}-\left(\ell_{4}^{\prime}+\ell_{3}\right) \tau=0, \\
\left(\ell_{4} \tau\right)^{(3)}-\left(\ell_{3} \tau\right)^{\prime \prime}+\left(\ell_{2} \tau\right)^{\prime}+4\left(\ell_{4} \tau^{\prime}\right)^{\prime \prime}-3\left(\ell_{3} \tau^{\prime}\right)^{\prime}+2 \ell_{2} \tau^{\prime} \\
\quad-\left(3 \ell_{1}+\ell_{2}^{\prime}\right) \tau+3 \ell_{3} \sigma-6\left(\ell_{4} \sigma\right)^{\prime}=0, \\
\left(\ell_{4} \sigma\right)^{(3)}-\left(\ell_{3} \sigma\right)^{\prime \prime}+\left(\ell_{2} \sigma\right)^{\prime}-\ell_{1} \sigma=0 .
\end{gathered}
$$

Unlike the case for $N=2$, we cannot uncouple these three moment equations unless $\tau \equiv 0$.

When $\tau \equiv 0$, H. L. Krall [7] classified all fourth-order differential equations having OP's as solutions.

Example 3.3. In [5], Koekoek and Meijer introduced the generalized Laguerre polynomials $\left\{L_{n}^{\alpha, M, N}(x)\right\}_{n=0}^{\infty}$, which are orthogonal relative to the positivedefinite Sobolev inner product:

$$
\phi(p, q)=\frac{1}{\Gamma(\alpha+1)} \int_{0}^{\infty} p(x) q(x) x^{\alpha} e^{-x} d x+M p(0) q(0)+N p^{\prime}(0) q^{\prime}(0) \text {, }
$$

where $\alpha>-1, M \geq 0$, and $N \geq 0$.

Note that $\phi(\cdot, \cdot)$ in equation (3.32) is exactly of the form (2.7), where $\sigma=$ $\frac{1}{\Gamma(\alpha+1)} H(x) x^{\alpha} e^{-x}+M \delta(x)$ is positive-definite, $\tau=N \delta(x)$ is discrete, and $H(x)$ is the Heaviside step function. Later, Koekoek [3, 4] found an infinite-order differential equation with polynomial coefficients satisfied by $\left\{L_{n}^{\alpha, M}, N(x)\right\}_{n=0}^{\infty}$. Furthermore, he also found differential equations of order $\leq 4 \alpha+10$ of type (1.1) satisfied by $\left\{L_{n}^{\alpha, M, N}(x)\right\}_{n=0}^{\infty}$ for $\alpha=0,1,2$. For $N>0$ and $\alpha=0,1$, or $2,\left\{L_{n}^{\alpha, M, N}(x)\right\}_{n=0}^{\infty}$ is the first example of an SOPS which is not an OPS and satisfies a differential equation of type (1.1).

Remark 3.5. It can be shown (see Lemma 0.1 in [8]) that for any PS $\left\{P_{n}(x)\right\}_{n=0}^{\infty}$ and any sequence of real numbers $\left\{\lambda_{n}\right\}_{n=0}^{\infty}$ with $\lambda_{0}=0$ and $\lambda_{m} \neq \lambda_{n}$ for $m \neq$ $n$, there exists a unique sequence $\left\{\ell_{i}(x)\right\}_{i=1}^{\infty}$ of polynomials with $\operatorname{deg}\left(\ell_{i}\right) \leq i$, $i \geq 1$, such that

$$
L\left[P_{n}\right](x)=\sum_{i=1}^{\infty} \ell_{i}(x) P_{n}^{(i)}(x)=\lambda_{n} P_{n}(x), \quad n \geq 0 .
$$




\section{ACKNOWLEDGMENT}

The second author was partially supported by Global Analysis Research Center during this research. The authors are sincerely grateful to the referee for many valuable comments.

\section{REFERENCES}

1. R. P. Boas, The Stieltjes moment problem for functions of bounded variation, Bull. Amer. Math. Soc. 45 (1939), 399-404.

2. A. J. Duran, The Stieltjes moment problem for rapidly decreasing functions, Proc. Amer. Math. Soc. 107 (1989), 731-741.

3. R. Koekoek, The search for differential equations for orthogonal polynomials by using computers, Delft University of Technology, Report no. 91-55, 1991.

4. _ The search for differential equations for certain sets of orthogonal polynomials, J. Comp. Appl. Math. 49 (1993), 111-119.

5. R. Koekoek and H. G. Meijer, A generalization of Laguerre polynomials, SIAM J. Math. Anal. 24 (1993), 768-782.

6. H. L. Krall, Certain differential equations for Tchebycheff polynomials, Duke Math. J. 4 (1938), 705-719.

7. _ On orthogonal polynomials satisfying a certain fourth order differential equation, The Penn State College Studies, no. 6, The Pennsylvania State College, PA, 1940.

8. H. L. Krall and I. M. Sheffer, Differential equations of infinite order for orthogonal polynomials, Ann. Mat. Pura Appl. 4 (1966), 135-172.

9. K. H. Kwon and L. L. Littlejohn, Classification of Sobolev orthogonal polynomials satisfying second order differential equations, RIM-GARC Preprint series 93-26, Seoul National Univ., 1933.

10. K. H. Kwon, L. L. Littlejohn, and B. H. Yoo, Characterization of orthogonal polynomials satisfying differential equations, SIAM J. Math. Anal. 25 (1994), 976-990.

11. L. L. Littlejohn and D. Race, Symmetric and symmetrisable ordinary differential expressions, Proc. London Math. Soc. 3(60) (1990), 344-364.

(I. H. Jung, K. H. Kwon, and D. W. Lee) Department of Mathematics, KAIST, 373-1 Kusong-DONG, Yusong-KU, TAEJon 305-701, Korea

E-mail address: khkwon@jacobi.kaist.ac.kr

(L. L. Littlejohn) Department of Mathematics and Statistics, Utah State University, LOGAN, UTAH 84322-3900

E-mail address: lance@sunf s.math.usu.edu 